Yüzüncü Yil Üniversitesi
Tarim Bilimleri Dergisi

Araştırma Makalesi (Research Article)

An Ethnobotanical Study of Medicinal Plants Used in Hizan District (Bitlis-Turkey) İbrahim DEMİR ${ }^{* 1}$

${ }^{1}$ Bitlis Eren University, Department of Biology, Faculty of Science, 13100, Bitlis, Turkey ${ }^{1}$ https://orcid.org/0000-0003-1533-556X

*Corresponding Author e-posta: idemir@beu.edu.tr

\section{Article Info}

Received: 30.04.2020

Accepted: 10.07.2020

Online Published 31.12.2020

DOI: $10.29133 /$ yyutbd.730202

\section{Keywords}

Bitlis,

Ethnobotany,

Hizan,

Medicinal plants.

\begin{abstract}
Medicinal plant use culture is very rich in East Anatolia. Hizan district is one of the best examples of this. Hizan has a very hilly topography. In the past, people is living in Hizan developed alternative medicines to treat their illnesses because of geographical structure and harsh winter conditions. This study was conducted in Hizan (Bitlis) district and it was the first investigation of the knowledge of tra-ditional medicinal plants used in Bitlis Province. This research aims to scientifically identify the medicinal plants used by local people and record the culture of traditional medicinal plants use of local people living in Hizan. Face-to-face interviews were conducted with participants with ethnobotanical knowled-ge and experience in 2018 and 2019 and the collected samples were prepared according to herbarium techniques. Consequently 71 taxa belonging to 29 families used for the treatment of 35 different diseases were identified. The traditional medical use of some taxa was specific to Hizan. The results revealed that the taxonomic family with the greatest number of utilized plants was Asteraceae. These are followed by Rosaceae, Lamiaceae, Malvaceae, Polygonaceae and Apiaceae.
\end{abstract}

\title{
Hizan İlçesinde Kullanılan Tıbbi Bitkilerin Etnobotanik İncelemesi (Bitlis-Türkiye)
}

\section{Makale Bilgileri}

Geliş: 30.04 .2020

Kabul: 10.07 .2020

Online Yayınlanma 31.12.2020

DOI: 10.29133 /yyutbd.730202

\section{Anahtar kelimeler}

Bitlis,

Etnobotanik,

Hizan,

Şifalı bitkiler.
Öz: Doğu Anadolu'da tibbi bitki kullanım kültürü çok zengindir. Hizan ilçesi bunun en güzel örneklerinden biridir. Hizan, oldukça engebeli bir topografyaya sahiptir. Geçmişte, Hizan halkı coğrafi yapısı ve sert kış koşulları nedeniyle hastalıklarını tedavi etmek için alternatif ilaçlar geliştirdi. Hizan (Bitlis) ilçesinde yürütülen bu çalışma Bitlis ilinde kullanılan geleneksel tıbbi bitkilerin bilgisinin ilk araştırmasıdır. $\mathrm{Bu}$ araştırma, yerel halk tarafından kullanılan tıbbi bitkileri bilimsel olarak tanımlamayı ve Hizan'da yaşayan yerel halkın geleneksel tıbbi bitki kullanım kültürünü kaydetmeyi amaçlamaktadır. 2018 ve 2019 yıllarında etnobotanik bilgi ve deneyime sahip katılımcılar ile yüz yüze görüşmeler yapılmış ve toplanan örnekler herbaryum tekniklerine göre hazırlanmıştır. Sonuç olarak 35 farklı hastalığın tedavisinde kullanılan 29 familyaya ait 71 takson tespit edilmiştir. Bazı taksonların geleneksel tıbbi kullanımı Hizan'a özgüdür. Sonuçlar, en fazla kullanılan bitki sınıfına sahip taksonomik familyanın Asteraceae olduğunu ortaya koymuştur. Bunları Rosaceae, Lamiaceae, Malvaceae, Polygonaceae ve Apiaceae takip etmiştir. 


\section{Introduction}

The fossil records show that the human use of plants as medicines may be traced back at least 60000 years (Solecki, 1975). According to some researchers (Miara et al., 2019), the great majority of developing countries (80\%) use medicinal plants for health purposes. Documentation of ethnobotanical information is important for the conservation and use of biological resources (Muthu et al., 2006).

Medicinal plants in rural areas of developing countries have been used as the primary source of medicine in the treatment of human diseases (Palombo, 2009; Sönmez et al., 2019).

Many ancient civilizations had flourished in Turkey, and thus many food and medicinal plant domestication began in this area (Altundağ and Öztürk, 2011). The Anatolian people have been used plants for medicinal purposes by Paleolithic period (50 000 BC). The plant specimens that were found in the Neanderthal tombs in Şanidar cave in the Zagros Mountains of northwestern Iraq, which is not far from Hizan (our research area), are solid proofs of this assumption (Baytop, 1999). Anatolia is the meeting place of three phytogeographical regions (Euro-Siberian, Mediterranean and lrano-Turanian). Thus, it has a very rich flora (Özhatay et al., 2005).

There are some ethnobotanical studies done in Eastern Anatolia, but they are inadequate (Akan et al., 2008). In the past, due to harsh winter conditions and deficiency, people who had difficulty accessing health facilities started looking for alternative medicines for treatment in Bitlis province. Thus, the use of the culture of the traditional medicinal plants has enriched. (Tabata et al., 1994). One of the best examples of this condition is Hizan district.

This study was carried out to scientifically identify the medicinal plants used by local people living in Hizan district, to record this cultural heritage and to convey it to future generations.

\section{Materials and Methods}

\subsection{The study area}

Hizan is located in Eastern Anatolia Region. It is located at $38^{\circ} 13^{\prime} \mathrm{N}, 42^{\circ} 25^{\prime} \mathrm{E}$ coordinates and is 1700 meter above average sea level. Hizan is neighbour to Bahçesaray and Gevaş (Van) in the east, Pervari and Şirvan (Siirt) in the south and Tatvan (Bitlis) in northwest (Figure 1).

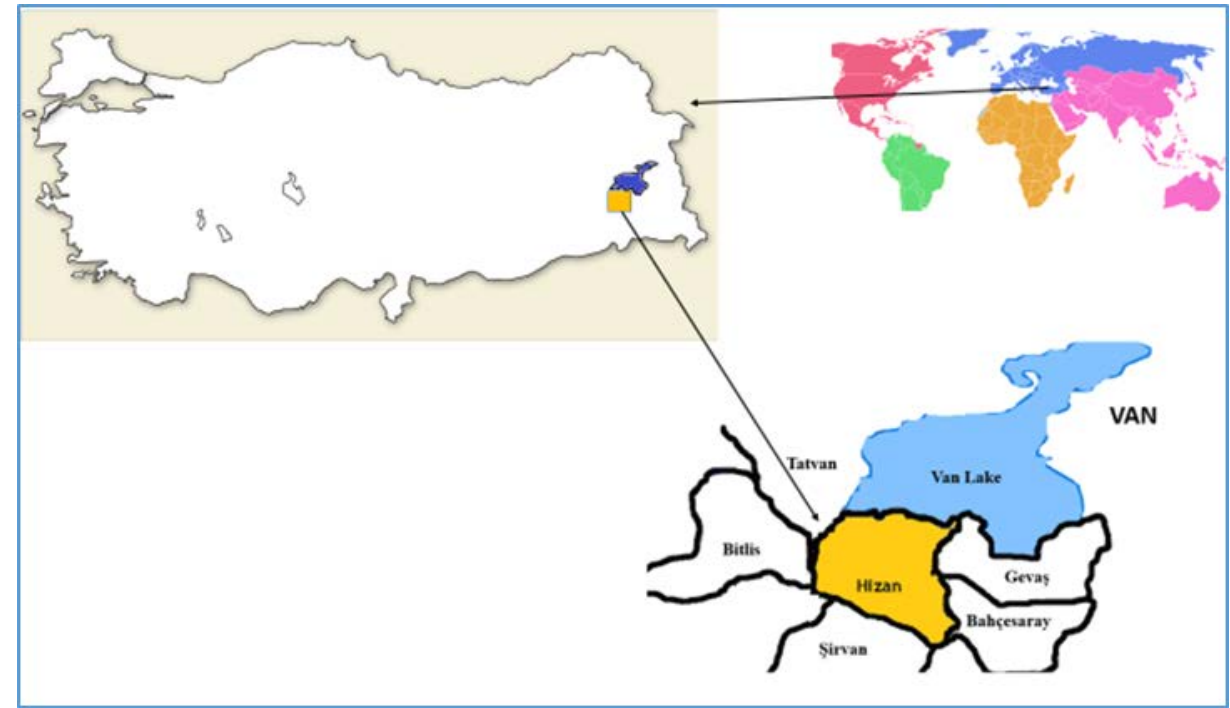

Figure 1. Geographical location of the research area (Hizan).

Hizan has been a center of civilization since the Hittites. According to historical records, human settlements are known to be present there from the 2000s BC. Human settlements started with the Hittites and continued under Persian, Roman, Byzantine and Arab rule. It was ruled by Seljuks in the 11th century and by Ottomans period in the 16th century (Çiçek, 2016). 


\subsection{Interviews with healer people}

In 2018 and 2019, interviews were held face-to-face with participants who have ethnobotanical knowledge and experience (Figure 2). The view was obtained from 68 people, including 39 men and 29 women and information was obtained recorded. The average age of the participants is over 40 . The job of these people living in the villages are generally farming.

The following questions were asked to the participants and the answers were recorded.

-What is the domestic names of the plant used?

-For what kind of diseases are the plants used?

- Which part of the plant is used?

- How you prepare a plant for use?

- How is used the herbal medicine?

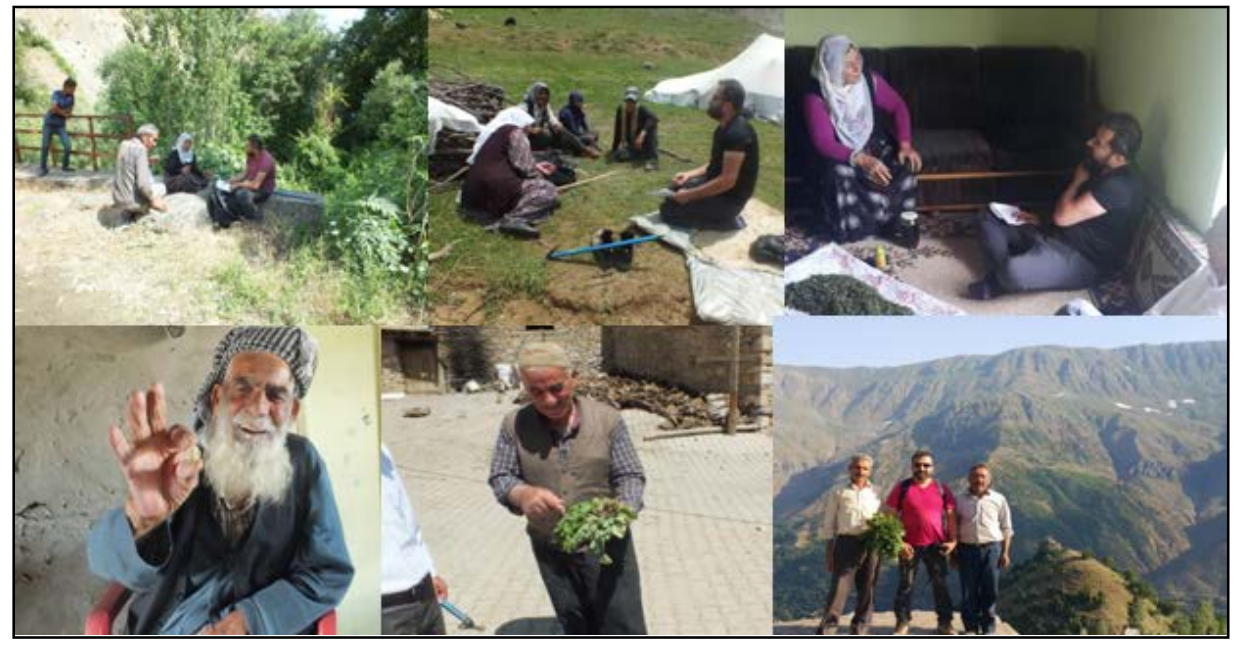

Figure 2. Geographical location of the research area (Hizan).

\subsection{Collecting and identification of medicinal plants}

Medicinally used plant samples were collected from their natural habitats with the participants. The collected plant samples were collected according to the information provided by the healers.

The collected samples were prepared according to herbarium techniques and herbarium samples were stored in the Biology Department of Faculty of Sciences, Bitlis Eren University.

Identification of plant taxa was performed by using Flora of Turkey. (Davis, 1965-1985; Davis et al., 1988; Güner et al., 2000). The scientific names of the plant taxa were confirmed by using (The Plant List, 2013) and The taxonomic categories (family, species etc.) of the identified samples were arranged in alphabetical order (Table 1).

\subsection{Classification of diseases}

The treated diseases were classified according to the international classifications of diseases of the by the WHO (Staub et al., 2015). These ailments are: Antidote; (for snake bites and bites of other venomous animals); Aphrodisias; (stimu-lant, anti-stimulant, impotency); Cancer, Cardiovascular; (anti-arrhythmic, cardiotonic, dropsy, blood circulation, haemorrhoids and varicose veins [both internal applications], hypertension); Dermatologic disorders;(incl. İnfections, tonsillitis, mouth and throat infection, haemorrhoids and varicose veins, external applications),Metabolic syndro-mes; (diabetes, immunological problems), Gastrointestinal problems; (Ulcers, stomac-he ache), Gynaecology (women's medicine), Infections (protozoal, bacterial and viral infections), Nervous system (peripheral and central, incl. Headache and insomnia, anal-gesics and unspecific spasmolytics, stimulants and hallucinogens), Parasites; (helminths, lice, insects); Respiratory complaints; (tonsillitis, mouth and 
throat infection, asthma, pulmonary problems), Skeleto-muscular system; (rheumatism, muscle relaxant, gout) and Urological problems; (infections, dropsy, prostate, diuretic action). shown above.

Herbal remedies prepared by the participants were arranged according to the diseases categories

\section{Results and Discussion}

\subsection{Taxonomic identification of plants}

The scientific name and voucher number, family name, local names, plant parts used of plants, preparation methods, utilization and uses of medicinal plants used in Hizan are given in Table 1.

As a result of the study, 71 taxa relating to 29 families that are used 735ort he treatment of 35 different diseases were detected.

The results revealed that the taxonomic family with the greatest number of utilized plants was Asteraceae (10 species). This is followed closely by Rosaceae, (9 species), and then comes Lamiaceae (5 species), Malvaceae, Polygonaceae and Apiaceae (4 species each), Boraginaceae and Plantaginaceae (3 each). The remaining plant families were represented by only one or two taxa.

The medical use of 8 taxa listed in Table 1 was first encountered in Hizan district. These taxa are; Paronychia kurdica subsp. Kurdica Boiss. (wart treanment), Rumex angustifolius subsp. Macranthus (Boiss.) Rech.f. (cancer), Scorzonera veratrifolia Fenzl (cancer, diabetes), Crataegus $x$ sinaica Boiss.( Cough, colds and flu), Satureja macrantha C.A.Mey. (Stomachache), Scutellaria orientalis subsp. Porphyrostegia J.R.Edm. (Hemorrhoids), Eryngium thyrsoideum Boiss. (Incision), Salix acmophylla Boiss. (Menstrual irregularities).

Scutellaria orientalis subsp. porphyrostegia J.R.Edm. was detected to be endemic plant used for medicinal purpose from Hizan. When compared withsome other studies conducted in the Eastern Anatolia region (Tabata et al., 1994; Altundağ and Öztürk, 2011; Kaval et al., 2014; Mükemre et al., 2015, Polat, 2019), it is seen that the local names of some commonly used plants are almost the same. Malva neglecta Wallr. (tolık, tolk, tollık), Rheum ribes L. (Ribes, ribes, rewas, ribez), E. Billardierei F.Delaroche (Tüsü), Urticadioica L. (Geznık, gezıng, gezgezok, gezne), Allium cepa L. (Pivaz, piyaz), Allium sativum L. (Sir) are some examples of this. However, some locally used names are specific to Hizan. The local names of plants species are: Asparagus persicus Baker (T1rhé mahré), Arctium tomentosum Mill. (Belge tehl), Chrysophthalmum montanum (DC.) Boiss. (Meryemxur), Scorzonera latifolia(Fisch. \& C.A.Mey.) DC. (Něrebend), Bryonia multiflora Boiss. \& Heldr. (Ture Gur), Quercus brantii Lindl. (Berüyè Hırçè), Paronychia kurdica subsp. Kurdica var. Kurdica Boiss (Giyayè tüyan), Satureja macrantha C.A.Mey. (Reyhana çiya), Scutellaria orientalis subsp. Porphyrostegia J.R.Edm. (Giyaye bavesirè), Hypericum scabrum L. (B1zın bezo), Teucrium polium L.(Cehdet), Rumex angustifolius subsp. Macranthus (Boiss.) Rech. F. (Bèjek), Potentilla recta L. (Z1la sor), Rosa canina L. (Masürük), Daphne mucronata Royle (Turi), Daucus carota L. (Giyaye Dıran ėş). These names are only used in Hizan (Table 1).

Some local names of the plants are characterized by the disease treated. For instance;" Giyaye bavesire " is used for Teucrium chamaedrys L. And Scutellaria orientalis subsp. porphyrostegia J.R.Edm. "Giya” means herb in Kurdish and "bavesir" is the name of the hemorrhoid disease.

Table 1. Database of ethnomedicinal plants of Hizan District

\begin{tabular}{|c|c|c|c|c|c|}
\hline $\begin{array}{l}\text { Family, Plant species, Collector } \\
\text { number }\end{array}$ & Local names & Part used & Preparation & $\begin{array}{l}\text { Utilization } \\
\text { method }\end{array}$ & Use \\
\hline \multicolumn{6}{|l|}{ AMARYLLIDACEAE } \\
\hline *Allium cepa L. İ.D. 2128 & Pivaz, piyaz & Bulb & Boiled & Ointment. & $\begin{array}{l}\text { Ringworm } \\
\text { treatment }\end{array}$ \\
\hline *Allium sativum L. İ.D. 2149 & Sir & Bulb & Crushed & $\begin{array}{l}\text { Ex(on } \\
\text { face), Eat. }\end{array}$ & $\begin{array}{l}\text { Acne treatment, } \\
\text { hypertension }\end{array}$ \\
\hline
\end{tabular}


Table 1. Database of ethnomedicinal plants of Hizan District (continued)

\begin{tabular}{|c|c|c|c|c|c|}
\hline $\begin{array}{l}\text { Family, Plant species, Collector } \\
\text { number }\end{array}$ & Local names & Part used & Preparation & $\begin{array}{l}\text { Utilization } \\
\text { method }\end{array}$ & Use \\
\hline \multicolumn{6}{|l|}{ ANACARDIACEAE } \\
\hline $\begin{array}{l}\text { Pistacia khinjuk Stocks. İ.D. } \\
\text { 2100,2105, 2124,2138,2140,2144 }\end{array}$ & Kèzvan & Gum, Fruit & $\begin{array}{l}\text { Latex } \\
\text { Crushed }\end{array}$ & $\begin{array}{l}\text { a. Wound } \\
\text { b.İnternlly. }\end{array}$ & $\begin{array}{l}\text { a.Inflamed wounds } \\
\text { b. Gastric ulcer, } \\
\text { aphrodisiac }\end{array}$ \\
\hline \multicolumn{6}{|l|}{ ARACEAE } \\
\hline $\begin{array}{l}\text { Arum rupicola Boiss. İ.D. } \\
2146,2163\end{array}$ & Kari & Leaves & Cooked & Eat. & $\begin{array}{l}\text { Lowering } \\
\text { tapeworm in } \\
\text { children }\end{array}$ \\
\hline $\begin{array}{l}\text { ASPARAGACEAE } \\
\text { Asparagus persicus Baker. İ.D. } \\
2097 \\
\text { ASTERACEAE }\end{array}$ & Tirhē mahrē & Root & Crushed & Oinment & Rheumatism \\
\hline $\begin{array}{l}\text { Achillea schischkinii Sosn. İ.D. } \\
2098\end{array}$ & Pujan & Aerial parts & Infusion & İnternally & Diabetes \\
\hline Achillea tenuifolia Lam. İ.D. 2093 & Pujdar & Aerial parts & Boiled & Bath & \multirow{2}{*}{$\begin{array}{l}\text { Body fatigue } \\
\text { a. Diabetes, } \\
\text { shortness of } \\
\text { breath. } \\
\text { b. Body fatigue }\end{array}$} \\
\hline $\begin{array}{l}\text { Achillea vermicularis Trin. İ.D. } \\
2142,2166\end{array}$ & Pèşdang & Capitulum & Infusion & $\begin{array}{l}\text { a.İnternally } \\
\text { b. Bath }\end{array}$ & \\
\hline $\begin{array}{l}\text { Achillea biebersteinii Afan. İ.D. } \\
2155\end{array}$ & Pujdang & Aerial parts & Cooked & Eat & Infertility \\
\hline $\begin{array}{l}\text { Arctium tomentosum Mill. İ.D. } \\
2108,2169\end{array}$ & Belge tehl & Leaves & Crushed & Wound & Rheumatism \\
\hline $\begin{array}{l}\text { Chrysophthalmum montanum (DC.) } \\
\text { Boiss. İ.D. } 2174\end{array}$ & Meryemxur & Leaves & Chew & Chew & Shortness of breath \\
\hline *Helianthus tuberosus L. İ.D. 2119 & Sèvka bınerd & Tuber & Eaten raw & Eat & Diabetes \\
\hline $\begin{array}{l}\text { Onopordum carduchorum. İ.D. } \\
2159\end{array}$ & Tovė karè & Seed & Crushed & $\begin{array}{l}\text { Eaten with } \\
\text { honey }\end{array}$ & $\begin{array}{l}\text { Hemorrhoids, } \\
\text { diabetes }\end{array}$ \\
\hline $\begin{array}{l}\text { Scorzonera latifolia(Fisch. \& } \\
\text { C.A.Mey.) DC. İ.D. } 2154\end{array}$ & Něrebend & Root & Latex & Chew & Gastric ulcer \\
\hline $\begin{array}{l}\text { Scorzonera veratrifolia Fenzl. İ.D. } \\
2175 \\
\text { BORAGINACEAE }\end{array}$ & Něrebend & Root & Latex & Chew & Diabetes \\
\hline $\begin{array}{l}\text { Anchusa azurea var. azurea Mill. } \\
\text { İ.D. } 2106,2187\end{array}$ & Güzürok & Aerial parts & Cooked & Eat & Antidote \\
\hline $\begin{array}{l}\text { Anchusa azurea var. kurdica } \\
\text { (Guşul.) D.F. Chamb İ.D. } 2122\end{array}$ & Güzürok & Aerial parts & Cooked & Eat & Antidote \\
\hline $\begin{array}{l}\text { Echium italicum L. İ.D. } 2089 \\
\text { BRASSICACEAE }\end{array}$ & Gurız & Aerial parts & Cooked & Eat & Antidote \\
\hline *Brassica oleracea L. İ.D. 2112 & Kelem & Leaves & Boiled & Oinment & $\begin{array}{l}\text { Rheumatism, to } \\
\text { step bledding }\end{array}$ \\
\hline Sinapis arvensis L. İ.D. 2180 & Xerdel & Seed & Crushed & $\begin{array}{l}\text { Eaten with } \\
\text { honey }\end{array}$ & Aphrodisiac \\
\hline $\begin{array}{l}\text { CHENOPODIACEAE } \\
\text { Beta vulgaris L. İ.D. } 2113 \\
\text { CUCURBİTACEAE }\end{array}$ & Silk & Leaves & Decoction & İnternally & Cholesterol \\
\hline $\begin{array}{l}\text { Bryonia multiflora Boiss. \& Heldr. } \\
\text { İ.D. } 2157\end{array}$ & Ture Gur & Fruit & Crushed & Oinment & Rheumatism \\
\hline $\begin{array}{l}\text { *Cucurbita maxima Lam. İ.D. } \\
2126 \\
\text { FABACEAE }\end{array}$ & Gundura şaş & Fruit & Boiled & Oinment & Anti-inflammatory \\
\hline $\begin{array}{l}\text { Astragalus gummifer Labill İ.D. } \\
2134\end{array}$ & Günü & Root & Gum & $\begin{array}{l}\text { Eaten with } \\
\text { honey }\end{array}$ & Liver diseases \\
\hline $\begin{array}{l}\text { *Medicago sativa L. İ.D. } 2168 \\
\text { FAGACEAE }\end{array}$ & Once & Leaves & Crushed & Oinment & to step bledding \\
\hline $\begin{array}{l}\text { Quercus infectoria Olivier İ.D. } \\
2167\end{array}$ & Mazü & Fruit & Crushed & $\begin{array}{l}\text { Apply as } \\
\text { powdered }\end{array}$ & Toothache \\
\hline Quercus brantii Lindl. İ.D. 2147 & Berüyė Hirçė & Fruit & Boiled & Eat & $\begin{array}{l}\text { Diabetes, Gastric } \\
\text { ulcer }\end{array}$ \\
\hline $\begin{array}{l}\text { HPERICACEAE } \\
\text { Hypericum scabrum L. İ.D. } 2162 \text {, } \\
2179\end{array}$ & Bizın bezo & Aerial parts & Crushed & Oinment & Incision \\
\hline
\end{tabular}


Table 1. Database of ethnomedicinal plants of Hizan District (continued)

\begin{tabular}{|c|c|c|c|c|c|}
\hline $\begin{array}{l}\text { Family, Plant species, Collector } \\
\text { number }\end{array}$ & Local names & Part used & Preparation & $\begin{array}{l}\text { Utilization } \\
\text { method }\end{array}$ & Use \\
\hline \multicolumn{6}{|l|}{ ILLECEBRACEAE } \\
\hline $\begin{array}{l}\text { Paronychia kurdica subsp. kurdica } \\
\text { var. kurdica Boiss. İ.D. } \\
\text { 2091,2130,2172 } \\
\text { JUNLANDACEAE }\end{array}$ & Giyayė tüyan & Aerial parts & Eaten raw & $\begin{array}{l}\text { Eat with } \\
\text { salt }\end{array}$ & Wart treatment \\
\hline *Juglans regia L. İ.D. 2111,2148 & $\begin{array}{l}\text { a. Pota güza } \\
\text { b. Xiça Guzè }\end{array}$ & $\begin{array}{l}\text { Fruit, } \\
\text { Sap }\end{array}$ & $\begin{array}{l}\text { a. Boiled } \\
\text { b. Sap }\end{array}$ & $\begin{array}{l}\text { a. İnternally } \\
\text { b. Drop }\end{array}$ & $\begin{array}{l}\text { a.Diabetes. } \\
\text { b. stomachache in } \\
\text { newborn child, ear } \\
\text { pain }\end{array}$ \\
\hline \multicolumn{6}{|l|}{ LAMIACEAE } \\
\hline $\begin{array}{l}\text { Origanum vulgare L. subsp. gracile } \\
\text { (K.Koch) Ietsw. İ.D. 2095, } 2110\end{array}$ & Cantıra reş & Aerial parts & Infusion & İnternally & $\begin{array}{l}\text { Shortness of } \\
\text { breath, enuresis }\end{array}$ \\
\hline $\begin{array}{l}\text { Satureja macrantha C.A.Mey. İ.D. } \\
2173,2181\end{array}$ & Reyhana çiya & Aerial parts & Crushed & İnternally & Stomachache \\
\hline $\begin{array}{l}\text { Scutellaria orientalis subsp. } \\
\text { porphyrostegia. İ.D. } 2177\end{array}$ & $\begin{array}{l}\text { Giyayè } \\
\text { bavesirè }\end{array}$ & Aerial parts & Infusion & İnternally & Hemorrhoids \\
\hline $\begin{array}{l}\text { Teucrium chamaedrys subsp. } \\
\text { sinuatum (Celak.) Rech. f. İ.D. } \\
\text { 2109,2186 }\end{array}$ & $\begin{array}{l}\text { Giyayè } \\
\text { bavesirè }\end{array}$ & Aerial parts & Infusion & İnternally & Hemorrhoids \\
\hline Teucrium polium L. I.D. 2092, 2129 & $\begin{array}{l}\text { 1.Meryemkot } \\
\text { 2.Cehdet }\end{array}$ & Leaves & $\begin{array}{l}\text { a. Eaten } \\
\text { raw } \\
\text { b. Infusion }\end{array}$ & $\begin{array}{l}\text { a. Eat } \\
\text { b. İnternally }\end{array}$ & $\begin{array}{l}\text { a. Diabetes b. } \\
\text { Afterpains }\end{array}$ \\
\hline \multicolumn{6}{|l|}{ MALVACEAE } \\
\hline $\begin{array}{l}\text { Alcea flavovirens(Boiss. \& Buhse) } \\
\text { Iljin. İ.D. } 2116\end{array}$ & Gûl & Root & Boiled & İnternally & Heart disease \\
\hline $\begin{array}{l}\text { Alcea remotiflora(Boiss. \& Heldr.) } \\
\text { Alef. İ.D. } 2117,2160\end{array}$ & Hèro & Aerial parts & Crushed & Hair washing & Hair care \\
\hline Malva neglecta İ.D. 2121, 2123 & $\begin{array}{l}\text { Tulık, Giya } \\
\text { dıpnu }\end{array}$ & Fruit & $\begin{array}{l}\text { a. Decoction } \\
\text { b. Cooked }\end{array}$ & $\begin{array}{l}\text { a. İnternally } \\
\text { b. Eat }\end{array}$ & $\begin{array}{l}\text { a. Stomachache b. } \\
\text { Afterpains }\end{array}$ \\
\hline $\begin{array}{l}\text { Malva slyvestris L. İ.D. } 2156 \\
\text { MORACEAE }\end{array}$ & Tulık & Aerial parts & Decoction & İnternally & Gastric ulcer \\
\hline $\begin{array}{l}\text { *Ficus carica subsp. rupestris } \\
\text { (Hausskn.) Browicz İ.D. } 2161 .\end{array}$ & Hejira reş & Bark & Crushed & Sniff & Nosebleed \\
\hline *Morus nigra L. İ.D. 2132,2143 & Tüye reş & Fruit & Crushed & $\begin{array}{l}\text { a. Drop } \\
\text { b. Eat }\end{array}$ & $\begin{array}{l}\text { a. Eye pain, redout } \\
\text { b. Icterus }\end{array}$ \\
\hline $\begin{array}{l}\text { PLANTAGINACEAE } \\
\text { Plantago lanceolata L. İ.D. 2087, } \\
2120\end{array}$ & Giyabirin & Aerial parts & $\begin{array}{l}\text { a. Eaten raw } \\
\text { b. Crushed }\end{array}$ & $\begin{array}{l}\text { a. Eat } \\
\text { b. wound }\end{array}$ & $\begin{array}{l}\text { a. Stomachache } \\
\text { b.to step bledding }\end{array}$ \\
\hline $\begin{array}{l}\text { Plantago major subsp. intermedia } \\
\text { (Gilib.) Linge İ.D. 2088,2102,2107 }\end{array}$ & $\begin{array}{l}\text { Takèş, } \\
\text { Heyiso }\end{array}$ & Aerial parts & $\begin{array}{l}\text { a. Decoction } \\
\text { b. Crushed }\end{array}$ & $\begin{array}{l}\text { a. İnternally b. } \\
\text { Externally }\end{array}$ & $\begin{array}{l}\text { a. Stomachache } \\
\text { b.Inflamed } \\
\text { wounds }\end{array}$ \\
\hline $\begin{array}{l}\text { PLATACEAE } \\
\text { Platanus orientalis L. İ.D. } 2086 \\
\text { POACEAE }\end{array}$ & Çınar & Leaves & Infusion & İnternally & Rheumatism \\
\hline *Zea mays L. İ.D. 2115 & Bacük & $\begin{array}{l}\text { Tassel of } \\
\text { corn }\end{array}$ & Decoction & İnternally & Kidney disease \\
\hline $\begin{array}{l}\text { POLYGONACEAE } \\
\text { Rheum ribes L. İ.D. } 2139,2143 \\
\text { Rumex angustifolius subsp. }\end{array}$ & Ribès & Root & Boiled & İnternally & Diabetes \\
\hline $\begin{array}{l}\text { macranthus (Boiss.) Rech. f. İ.D. } \\
2188\end{array}$ & Bèjek & Aerial parts & Cooked & Eat & Cancer \\
\hline Rumex crispus L. İ.D. 2085 & Avilok & Fruit & Crushed & Oinment & Inflamed wounds \\
\hline Rumex patientia L. İ.D. 2118 & Avilok & Aerial parts & $\begin{array}{l}\text { a. Decoction. } \\
\text { b. Crushed }\end{array}$ & $\begin{array}{l}\text { a. İnternally } \\
\text { b. Oinment }\end{array}$ & $\begin{array}{l}\text { a. Intestinal ulcer } \\
\text { b.to stop bledding }\end{array}$ \\
\hline RANUNCULACEAE & & & & & \\
\hline *Nigella sativa L. İ.D. 2096 & Çörek otu & Seed & Crushed & $\begin{array}{l}\text { Eat with } \\
\text { honey }\end{array}$ & Cancer \\
\hline $\begin{array}{l}\text { Ranunculus kotschyi Boiss. İ.D. } \\
2135\end{array}$ & Çüng & Aerial parts & Eaten raw & Eat & Cancer \\
\hline
\end{tabular}


Table 1. Database of ethnomedicinal plants of Hizan District (continued)

\begin{tabular}{|c|c|c|c|c|c|}
\hline $\begin{array}{l}\text { Family, Plant species, Collector } \\
\text { number }\end{array}$ & Local names & Part used & Preparation & $\begin{array}{l}\text { Utilization } \\
\text { method }\end{array}$ & Use \\
\hline \multicolumn{6}{|l|}{ ROSACEAE } \\
\hline $\begin{array}{l}\text { Amygdalus orientalis Miller. İ.D. } \\
2158\end{array}$ & Bihèv & Seed & Eaten raw & $\begin{array}{l}\text { Eat with } \\
\text { honey }\end{array}$ & $\begin{array}{l}\text { Diabetes, } \\
\text { headache }\end{array}$ \\
\hline $\begin{array}{l}\text { Crataegus meyeri Pojark. İ.D. } \\
2151\end{array}$ & $\begin{array}{l}\text { Reyě gühüja } \\
\text { spi }\end{array}$ & Root & Decoction & İnternally & Tuberculosis \\
\hline $\begin{array}{l}\text { Crataegus x sinaica Boiss. İ.D. } \\
2190\end{array}$ & Gühüj & Fruit & Infusion & İnternally & $\begin{array}{l}\text { Cough, colds and } \\
\text { flu }\end{array}$ \\
\hline $\begin{array}{l}\text { Crataegus curvisepala Lindm. İ.D. } \\
2176\end{array}$ & Reyè gühüjè & Root & Boiled & İnternally & Tuberculosis \\
\hline $\begin{array}{l}\text { Malus sylvestris subsp. orientalis } \\
\text { var. orientalis (Uglitzk.) Browicz. } \\
\text { İ.D. } 2103,2133\end{array}$ & Sèva gevr & Fruit & Cooked & Eat & Tuberculosis \\
\hline Potentilla recta L. İ.D. 2182 & Zila sor & Aerial parts & Boiled & Oinment & Rheumatism \\
\hline $\begin{array}{l}\text { Rosa canina L. İ.D. } \\
2104,2125,2137\end{array}$ & Masürük & Fruit & Decoction & İnternally & $\begin{array}{l}\text { Cough, colds, flu } \\
\text { and stomachache }\end{array}$ \\
\hline Rosa puberulenta Rydb. İ.D. 2191 & Masürük & Fruit & Decoction & İnternally & $\begin{array}{l}\text { Cough, colds and } \\
\text { flu }\end{array}$ \\
\hline $\begin{array}{l}\text { Rubus idaeus L. İ.D. } 2153 \\
\text { SALİCACEAE }\end{array}$ & Tütürk & Fruit & Eaten raw & Eat & Blood thinner \\
\hline Salix acmophylla Boiss. İ.D. 2152 & Dara biyě & Leaves & Decoction & İnternally & $\begin{array}{l}\text { Menstrual } \\
\text { irregularities }\end{array}$ \\
\hline $\begin{array}{l}\text { SCROPHULARIACEAE } \\
\text { Verbascum oreophilum var. } \\
\text { joannis. (Bordz.) Hub.-Mor. İ.D. } \\
2184\end{array}$ & Sinem & Aerial parts & Boiled & Bath. & Diabetes \\
\hline $\begin{array}{l}\text { THEACEAE } \\
\text { Daphne mucronata Royle. İ.D. } \\
2189 \\
\text { TILIACEAE }\end{array}$ & Turi & Fruit & Crushed & Oinment & Rheumatism \\
\hline Tilia tomentosa Moench İ.D. 2094 & Ihlamur & Flower & Infusion & İnternally & $\begin{array}{l}\text { Cough, colds and } \\
\text { flu }\end{array}$ \\
\hline \multicolumn{6}{|l|}{ UMBELLIFFERAE } \\
\hline Daucus carota L. İ.D. 2101 & $\begin{array}{l}\text { Giyayė Dıran } \\
\text { èş }\end{array}$ & & Crushed & Mouthwash & Toothache \\
\hline Eryngium billardierei F. İ.D. 2136 & Tüsü & $\begin{array}{l}\text { Fresh } \\
\text { shoots }\end{array}$ & Crushed & Oinment & Burns \\
\hline $\begin{array}{l}\text { Eryngium pyramidale Boiss. \& } \\
\text { Hausskn., İ.D. } 2165\end{array}$ & Tüsü & Root & Decoction & İnternallt & Shortness of breath \\
\hline $\begin{array}{l}\text { Eryngium thyrsoideum Boiss. İ.D. } \\
2170\end{array}$ & Xiça tüsü & $\begin{array}{l}\text { Fresh } \\
\text { shoots }\end{array}$ & Crushed & Powder & Incision \\
\hline $\begin{array}{l}\text { *Petroselinum crispum (Mill.) } \\
\text { A.W.Hill. İ.D. } 2114\end{array}$ & Maydanoz & Root & Decoction & İnternally & Kidney diseases \\
\hline $\begin{array}{l}\text { Prangos pabularia Lindl. İ.D. } \\
2178 \\
\text { URTICACEAE }\end{array}$ & Xiça heliz & Aerial parts & Ointment & Wound & Erysipeloid \\
\hline $\begin{array}{l}\text { Urtica dioica L. İ.D. 2099, } \\
2127,2141,2145,2164,2183\end{array}$ & Geznık & $\begin{array}{l}\text { Seed } \\
\text { Aerial parts } \\
\text { Root }\end{array}$ & Crushed & $\begin{array}{l}\text { a. Eatwith } \\
\text { honey } \\
\text { b. Oinment } \\
\text { c. Oinment }\end{array}$ & $\begin{array}{l}\text { a. aphrodisiac, } \\
\text { cancer } \\
\text { b. Rheumatism } \\
\text { c. bone fracture } \\
\text { treatment }\end{array}$ \\
\hline
\end{tabular}

\subsection{Parts used and preparation method}

Generally used plant parts are the following ones; Aerial part (32\%), Fruits (21\%), Leaves (15\%), Roots (15\%), Seeds (5\%). Other parts were represented by only one or two percent (total 17\%). (Figure 3). 


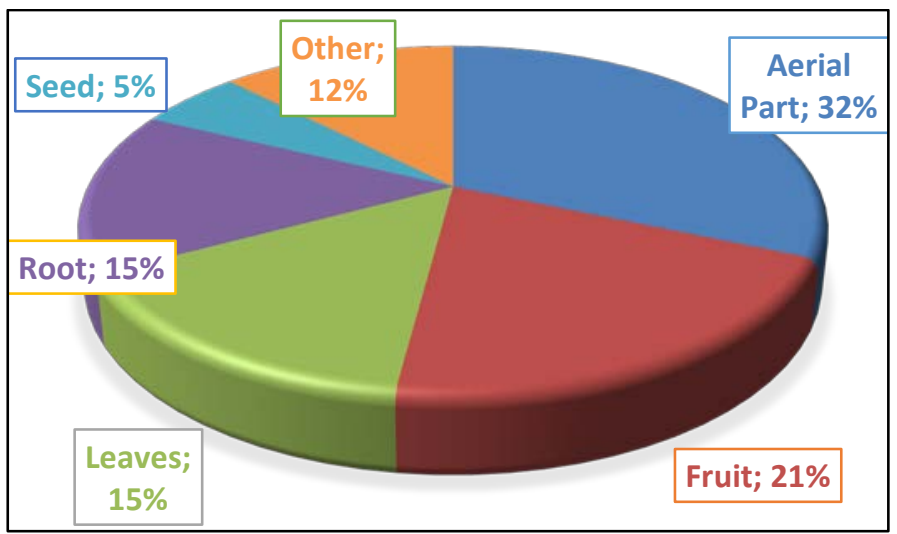

Figure 3. Parts of the plants used.

\subsection{Traditional medicinal plants used for the treatment of different diseases in hizan}

The plants used for types of diseases below are arranged according to the diseases classified by some authors (Staub et al., 2015). Dermatologic disorders (13), Metabolic syndromes (12), Gastrointestinal problems (11), Skeleto-muscular system disorders (10), Cardiovascular problems (10), Respiratory complaints (8), Nervous systemdiseases (5), Cancer (5), Infections (5), Gynecological diseases(4), Urological problems (3), Antidote (3), Aphrodisias (3) and Parasites (1).The reported ailments were grouped into 14 categories basing on the information gathered from the interviews (Figure 4).

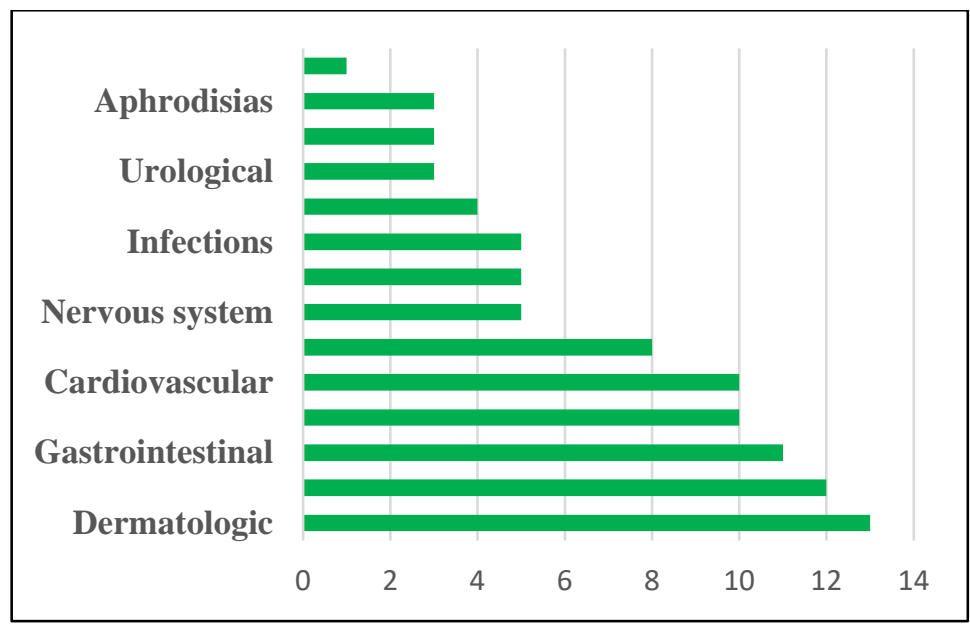

Figure 4. Number of taxa used for folk medicine in Hizan.

The most used plants in the research area are:

Urtica dioica L., (for aphrodisias, cancer and skeleto-muscular system) Achillea vermicularis Trin. (metabolic syndromes, respiratory complaints, skeleto-muscular system), Plantago lanceolata and Plantago major L. (dermatologic disorders, gastrointestinal problems).,Rheum ribes L. (diabetes), Pistacia khinjuk Stocks (dermatologic disorders, gastrointestinal problems and aphrodisias), Malva neglecta Wallr. (gastrointestinal problems and Gynaecology) Teucrium chamaedrys subsp sinuatum (Celak.) Rech.f. and Onopordum carduchorum Bornm. \& Beauverd (hemorrhoids), Anchusa azurea (for antidote) and Paronychia kurdica subsp. kurdica var. kurdica Boiss. (wart treatment) are most used species.

Urtica dioica L. is plants which are commonly used across the world and in Turkey. It is commonly used to treat cancers. In addition, this herb is used to diabetes disease, rheumatism, stomachache, colds, and flu arthritis, digestive, diuretic, genital disorders, in East Anatolia (Altunda $\breve{g}$ and Özturk, 2011; Mükemre et al., 2015). 
Plantago species (P. lanceolata L. and P. major L.) are widespread in Anatolia. They are used to treat abscess, gastric pain, hemorrhoids vulnerary, antiinflammatory, stomachic, ulcer, antihemorrhoidal, gynecologic diseaes, to cure wounds, embolism, urinary inflammations, to heal wounds, antitussive, bronchitis, diarrhoea, expectorant, urinary inflammations (Tabata et al.,1994; Kaval et al., 2014; Mükemre et al., 2015).

Rheum ribes L. is one of the most collected plants from natural areas in East Anatolia. Fresh shoots of this plant are consumed as food in Eastern Anatolia and they are used for the treatment of diabetes (Tabata et al.,1994; Altundağ and Öztürk, 2011; 2015; Mükemre et al., 2015).

Pistacia khinjuk Stocks has antibacterial, antifungal, antioxidant, and wound healing properties (Al-Alfy et al., 2019).

Pistacia khinjuk widely used both for the treatment of diseases and as nutrient in Hizan. Also a latex ointment made from the trunk of the tree is also applied to inflamed wounds. Pistacia khinjuk Stocks was used for stomachache in the study conducted in Hakkâri province (Kaval et al., 2014). P. khinjuk has been used as an indigestion, tonic, toothache and astringent folk medicine. In addition, fruits of it used edible wild fruits (Pirbalouti and Aghaee 2011).

The use of malva neglecta Wallr. is common in all regions of Turkey. It is commonly consumed as food. And it is commonly used as a folk medicine in a number of conditions. In Hizan it is widely used for the treatment of stomachache as a pain reliever.

Many species of Teucrium are known for their utilization in traditional folk medicine. They are claimed to exhibit interesting biological properties such as; hypoglycaemic, hypolipidemic, antioxidant, antipyretic, anti-inflammatory, antiulcer, antitumor and anti bacterial (Bruno et al., 2004).

Onopordum carduchorum Bornm. \& Beauverd is used forthe treatment of hemorrhoids in Hizan. Ethnobotanical use of this plant for the same disease is found in Savur district (Mardin). (Arasan and Kaya, 2015).

The study called "Effects of Paronychia kurdica Boiss. on teat and udder papillomatosis in cows" was investigated and successful results were obtained (Apaydin et al., 2010).

Ethnobotanical record of Rumex angustifolius subsp. macranthus (Boiss.) Rech.f. plants were not found.

\section{Conclusion}

Plants, which are of great importance for the continuity of life, are among the most im-portant natural resources of a country.

Plant-human relationship is as old as human history. Almost all civilizations have used plants as a mean of treatment. The culture of medicinal plant use is very rich in Anatolia. It has hosted many civilizations throughout history. In the past, people who lived in places like Hizan where they had difficulty in accessing health care facilities due to challenging geographical structuresand harsh winter conditions began to look for alternative reme-dies to treat their illnesses. Undoubtedly, the rich plant diversity in the region played an important role in alternative treatments. Ethnobotanical uses are gaining value dayby day. Unfortunately, the value of this knowledge is not known well enough by younger generations. The number of people who have this knowledge is decreasing day by day. Therefore, ethnobotanical knowledge has to be recorded as soon as possible. Ethnobotanical research is important because it contains valuable information that has been acquired by trial and error and has been passed down from generation to generation as a result of a long period of time. With this study, it was aimed to record the plants' use culture of the local people living in Hizan district which has rich plant diversity and plant use accumulation.

\section{Acknowledgment}

This study was carried out with funding provided by BEBAP (Office of Scentific Re-search Projects of Bitlis Eren University), Bitlis/Turkey (Project no: 2018.07).

\section{References}

Akan, H., Korkut, MM., \& Balos, MM. (2008). An ethnobotanical study around Arat Mountain and its surroundings (Birecik Sanliurfa). Firat University Journal of Engineering Sciences. 20, 67-81. 
Al-Alfy, N.S., Moustafa, A.R.M., Alotaibi, M.O., \& Mansour S.R. (2019). Impact of Climate Change on Pistacia khinjuk as a Medicinal Plant in Egypt and Saudi Arabia. Appl Sci Res Rev. 6(1), 3.

Altundağ, E., \& Özturk, M. (2011). Ethnomedicinal studies on the plant resources of east Anatolia, Turkey. Procedia-Social and Behavioral Sciences. 19, 756-777.

Apaydin, A.M., Aydin, M., Çiftçi, O., Timurkaan, N., Yildiz, H., \& Tonbak, S. (2010). Effects of Paronychia kurdica on teat and udder papillomatosis in cows. Revue de Medecine Veterinaire, 161(6), 267.

Arasan, S., \& Kaya, I. (2015). Some important plants belonging to asteraceae family used in folkloric medicine in Savur (Mardin/Turkey) area and their application areas. Journal Food Nutrition Research. 3, 337-340.

Baytop, T. (1999). Therapy with medicinal plants in Turkey (Past and Present),second ed. Istanbul: Nobel Medicine Publication.

Bruno, M., Rosselli, S., Maggio, A., Piozzi, F., Scaglioni, L., Arnold, N.A., \& Simmonds, M.S. (2004). Neoclerodanes from Teucrium orientale. Chemical and pharmaceutical bulletin. 52(12), 14971500.

Çiçek, H. (2016). Bir mekân Hizan: ilim ve âlim havzası. Katre Uluslararası Insan Araştırmaları Dergisi,1, 189-198.

Davis, P.H (1965-1985). Flora of Turkey and the East Aegean Islands, first edit. 1-9.Edinburgh: Edinburgh Univ. Press,

Davis, P.H., Mill, R.R., \& Tan, K. (1988). Flora of Turkey and the East Aegean Islands, first ed. 10.Edinburgh: Edinburgh Univ. Press.

Güner, A., Özhatay, N., Ekim, T., \& Başer, K. (2000). Flora of Turkey and the East Aegean Islands, first ed. 11.Edinburgh: Edinburgh Univ. Press.

Kaval, İ., Behçet, L., \& Çakılcıoğlu, U. (2014). Ethnobotanical study on medicinal plants in Geçitli and its surrounding (Hakkâri-Turkey). Journal of ethnopharmacology.155, 171-184

Miara, M.D., Bendif, H., Rebbas, K., Rabah, B., Hammou, M.A., \& Maggi, F. (2019). Medicinal plants and their traditional uses in the highland region of Bordj Bou Arreridj (Northeast Algeria). Journal of Herbal Medicine. 100262.

Muthu, C., Ayyanar, M., Raja, N., \& Ignacimuthu, S. (2006). Medicinal plants used by traditional healers in Kancheepuram District of Tamil Nadu, India. Journal of Ethnobiology and ethnomedicine, 2(1), 1-10.

Mükemre, M., Behçet, L., \& Çakılcıoğlu, U. (2015). Ethnobotanical study on medicinal plants in villages of Çatak (Van-Turkey). Journal of Ethnopharmacology, 166, 361-374.

Özhatay, N., Byfield, A., \& Atay, S. (2005). Türkiye’nin 122 Önemli Bitki Alanı. İstanbul: WWF.

Pirbalouti, A. G., \& Aghaee, K. (2011). Chemical composition of essential oil of Pistacia khinjuk stocks grown in Bakhtiari Zagross Mountains, Iran. Electronic Journal of Biology, 7(4), 67-69.

Palombo, E. A. (2009). Traditional medicinal plant extracts and natural products with activity against oral bacteria: potential application in the prevention and treatment of oral diseases. Evid-Based Complementary and Alternative Medicine, 2011, 1-15.

Polat, R. (2019). Ethnobotanical study on medicinal plants in Bingöl (City center)(Turkey). Journal of Herbal Medicine, 16, 100211.

Solecki, R. (1975). Shanidar IV, a Neanderthal flower burial in northern Iraq. Science, 190, 880-881.

Sönmez, E., Kırbağ, S., \& İnci, Ş (2019). Antifungal and antibacterial effect of dodder (Cuscuta campestris) used for hepatitis treatment of mothers and newborn infants in province Mardin in Turkey. Yüzüncü Yıl Üniversitesi Tarım Bilimleri Dergisi, 29(4), 722-730.

Staub, P.O., Geck, M.S., Weckerle, C.S., Casu, L., \& Leonti, M. (2015). Classifying diseases and remedies in ethnomedicine and ethnopharmacology. Journal of Ethnopharmacology, 174, 514 519.

Tabata, M., Sezik, E., Honda, G., Yeşilada, E., Fukui, H., Goto, K., \& Ikeshiro, Y. (1994). Traditional medicine in Turkey III. Folk medicine in East Anatolia, Van and Bitlis provinces. International Journal of Pharmacognosy, 32(1), 3-12.

The Plant List. (2019). http://www.theplantlist.org, (accessed 05 October 2019). 